\title{
USO E DIVERSIDADE DE PLANTAS MEDICINAIS EM SANTO ANTONIO DO LEVERGER, MT, BRASIL ${ }^{1}$
}

\author{
Maria Christina de Mello Amorozo ${ }^{2}$
}

Recebido em 23/11/1999. Aceito em 27/12/2001.

\begin{abstract}
RESUMO - (Uso e diversidade de plantas medicinais em Santo Antonio do Leverger, MT, Brasil). O presente trabalho tem como objetivo fazer o levantamento etnobotânico de plantas com usos terapêuticos no município de Santo Antonio do Leverger, MT, e estimar a diversidade de espécies usadas. Foram entrevistados residentes adultos de ambos os sexos, junto com os quais foram coletadas as plantas, depositadas no Herbarium Rioclarense (HRCB). Identificaram-se 228 espécies, pertencentes a 73 famílias; $56 \%$ delas crescem espontaneamente em ambientes naturais ou antropicamente modificados, $41 \%$ são cultivadas e 3\% são compradas. Os índices de diversidade encontrados comparam-se aos mais altos registrados na literatura para plantas medicinais em outras áreas tropicais. A riqueza e diversidade das plantas espontâneas podem espelhar até certo ponto a riqueza e diversidade de espécies no ambiente, enquanto tais parâmetros para as espécies cultivadas estariam mais ligados a fatores sócio-culturais que promovam a introdução de novas plantas e informações de uso a partir de fontes externas. Sugere-se que quando comunidades tradicionais se tornam mais expostas à sociedade nacional, o número de espécies e o conhecimento acerca de seu uso podem sofrer inicialmente um acréscimo, por aportes externos; mas, com o aprofundamento do contato, e as mudanças sócio-econômicas decorrentes, a tendência será que as plantas usadas com fins terapêuticos restrinjam-se às espécies cultivadas e invasoras cosmopolitas.
\end{abstract}

Palavras-chave - etnobotânica, plantas medicinais, índices de diversidade, Mato Grosso

ABSTRACT - (Use and diversity of medicinal plants in Santo Antonio do Leverger, MT, Brazil). This work describes an ethnobotanical survey of medicinal plants in Santo Antonio do Leverger Municipality, Mato Grosso State, Brazil and estimates the diversity of species with therapeutic use. Adult dwellers, male and female, were interviewed and plant gathering undertaken with their assistance. Voucher specimens were deposited at the Herbarium Rioclarense (HRCB). 228 species were identified, belonging to 73 families; 56\% of the species grow spontaneously in natural and anthropically modified habitats, $41 \%$ are cultivated and $3 \%$ are purchased. Diversity indexes are among the highest ones reported in literature for medicinal plants in other tropical areas. Richness and diversity of spontaneous species may mirror to certain extent environmental richness and diversity, whereas for cultivated species, these measures could be linked to socio-cultural factors enhancing introduction of new plants and use information from outside the area. It is suggested that when traditional communities become more exposed to society at large, species number and knowledge about their use may at first increase, by foreign input; but, with the socio-economic changes that come about over time, plants used for therapeutic aims will be limited to the cultivated species and cosmopolite weeds.

Key words - ethnobotany, medicinal plants, diversity indices, Mato Grosso State, Brazil

\footnotetext{
${ }^{1}$ Auxílio Pesquisa FAPESP.

${ }^{2}$ Departamento de Ecologia, IB, UNESP. C.Postal 199, CEP 13506-900, Rio Claro, São Paulo, Brasil.
} 


\section{Introdução}

Muitas sociedades tradicionais ou autóctones possuem uma vasta farmacopéia natural, em boa parte proveniente dos recursos vegetais encontrados nos ambientes naturais ocupados por estas populações, ou cultivados em ambientes antropicamente alterados.

O interesse acadêmico a respeito do conhecimento que estas populações detêm sobre plantas e seus usos tem crescido, após a constatação de que a base empírica desenvolvida por elas ao longo de séculos pode, em muitos casos, ter uma comprovação científica, que habilitaria a extensão destes usos à sociedade industrializada (Farnsworth 1988). Além disso, cada vez mais se reconhece que a exploração dos ambientes naturais por povos tradicionais pode nos fornecer subsídios para estratégias de manejo e exploração que sejam sustentáveis a longo prazo. Atualmente, vários autores têm proposto formas de se avaliar a interação destas populações com os recursos naturais de que dispõem. Begossi (1996), por exemplo, propõe a utilização de conceitos ecológicos, como o de diversidade de espécies, para auxiliar na compreensão das interações homem-ambiente; afirma que o emprego de índices de diversidade pode ser útil para comparar a exploração de recursos biológicos feita por diferentes populações humanas, com culturas diversas, ocupando diferentes ambientes. Outros autores propõem a elaboração de índices que possibilitem quantificar o uso de espécies ou famílias de plantas (por exemplo, Prance et al. 1987; Phillips e Gentry 1993 a, b), para avaliar sua importância como recurso para as populações locais. Estas ferramentas permitem estabelecer comparações tanto em relação à exploração dos recursos, quanto em relação às formas de seu uso, em diferentes locais e entre diferentes populações humanas.

Este trabalho tem por objetivos: a) fazer o levantamento etnobotânico das plantas com usos terapêuticos no Município de Santo Antonio do
Leverger, MT; b) calcular os índices de diversidade das espécies citadas; c) comparar com os índices encontrados para outros locais e discutir seu comportamento em relação a fatores ambientais e sócio-culturais.

\section{Material e métodos}

Área de estudo - Situa-se na Baixada Cuiabana, no Município de Santo Antonio do Leverger, MT, entre 20 e $30 \mathrm{~km}$ ao sul de Cuiabá, nas margens do rio Cuiabá e ao norte dos Pantanais Matogrossenses (Fig. 1). Seu clima apresenta duas estações bem definidas, uma chuvosa (outubro a março), e outra seca (abril a setembro) (Alvarenga et al 1984). As precipitações médias anuais ficam em torno dos 1500 a $1700 \mathrm{~mm}$ e as médias anuais de temperatura são elevadas $\left(23^{\circ}\right.$ a $25^{\circ} \mathrm{C}$, Brasil, Ministério das Minas e Energia 1982). A formação vegetal predominante é o cerrado (desde campo limpo até cerradão), apresentando floresta decídua na encosta dos morros e floresta de galeria ao longo dos rios, além de, em alguns trechos, vegetação típica de áreas alagadas.

As principais atividades econômicas são a agricultura familiar, a pesca, a fabricação de farinha de mandioca para auto-consumo e comercialização; algumas atividades ligadas ao turismo começam a ser implementadas. Nos anos recentes, as influências externas vêm se tornando marcantes, aprofundadas pela implantação de infra-estrutura moderna, que permite a rápida penetração na área da ideologia urbanoindustrial.

A coleta de dados foi feita em três comunidades rurais (Morro Grande, Barreirinho e Varginha) e na sede do município. Os dados foram coletados esporadicamente em várias ocasiões entre 1991 e 1994, e, sistematicamente, em fevereiro, março e junho de 1997, fevereiro, março, maio e junho de 1998. Utilizaram-se observação participante, entrevistas semiestruturadas e estruturadas (Bernard 1988), vi- 


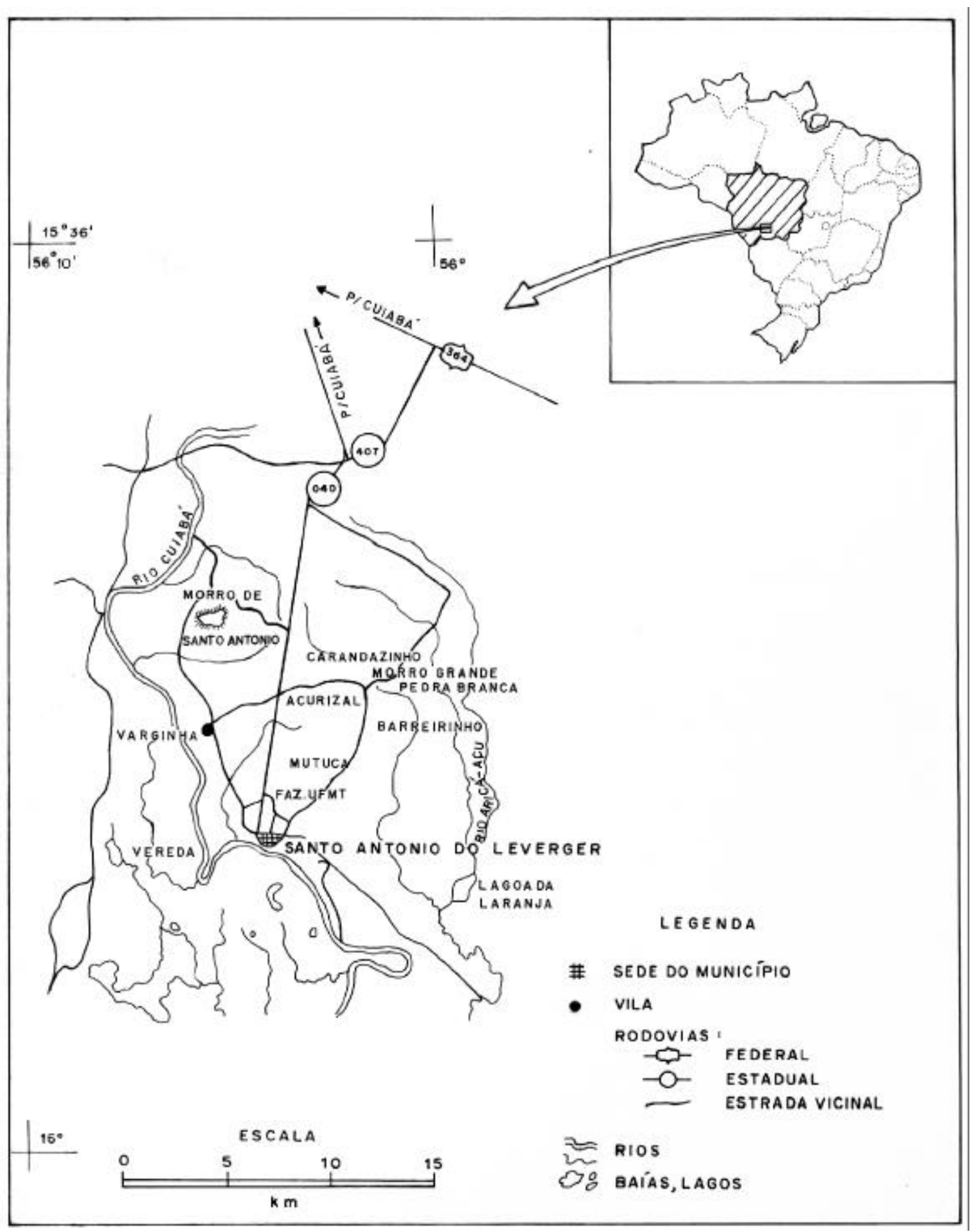

Figura 1. Localização da área de estudo. Fonte: Fundação de Recursos Cândido Rondon, 1991. 
sando a obtenção de características sócio-econômicas dos informantes, características botânicas e ecológicas das plantas usadas para fins medicinais e suas indicações terapêuticas.

Foram entrevistados vinte e quatro adultos, (quinze mulheres e nove homens), escolhidos com base no conhecimento com relação ao uso de plantas medicinais e procedimentos terapêuticos; com metade deles (quatro dos quais benzedores), manteve-se um contato prolongado, com entrevistas em várias ocasiões ao longo da pesquisa. Além disso, entrevistas e conversas ocasionais foram mantidas com cerca de mais vinte e quatro indivíduos. Os entrevistados têm idades acima de 50 anos, com exceção de duas mulheres, na faixa entre 30 e 40 anos. $O$ grau de escolaridade é baixo, em geral não ultrapassando as quatro primeiras séries do primeiro grau, sendo que cerca de $24 \%$ da amostra não têm nenhuma escolaridade ou são analfabetos funcionais. A maior parte está ou esteve, durante a maior parte de sua vida, ligada a atividades agrícolas e, em alguns casos, também à pesca.

As plantas foram coletadas dos ambientes onde crescem, na presença e por indicação dos informantes. Os espécimes prensados e secos foram identificados pelos seguintes taxonomistas: A.L. Prado, UFMT, Cuiabá, MT; M.A. Assis, UNESP, Rio Claro, SP; J.R. Pirani e A. Zanin, USP, São Paulo, SP; I. Cordeiro; M.C..H. Mamede, Instituto de Botânica, São Paulo, SP e L.C.Ming, UNESP, Botucatu, SP. As exsicatas estão depositadas no Herbarium Rioclarense (HRCB). Para algumas plantas domesticadas, a identificação foi feita no local e com o auxílio da literatura.

As espécies com aplicação terapêutica consideradas neste trabalho incluem, além daquelas indicadas para afecções que têm correspondência na medicina oficial, também aquelas espécies usadas para doenças e estados de desconforto que são identificados pela população local, mas não reconhecidos pela biomedicina, como, por exemplo, quebrante, inveja, feitiço, arca-caída, considerando-se que fazem parte do universo nosológico das comunidades estudadas (Bruneli 1987). As indicações foram agrupadas com base na classificação das doenças proposta pela Organização Mundial de Saúde (OMS 2000); as doenças ou estados que não puderam ser incluídos nesta classificação geral foram agrupados na categoria "doenças culturais".

Para se calcular diversidade, empregou-se o índice de Shannon-Wiener,

$$
\mathrm{H}^{\prime}=-\sum_{\mathrm{i}=1}^{\mathrm{s}}(\mathrm{pi})(\log \mathrm{pi})
$$

base 10 e $e$, onde: $\mathrm{s}=$ número de espécies $\mathrm{e}$

$$
\mathrm{pi}=\mathrm{n}_{\mathrm{i}} / \mathrm{N}, \mathrm{N}=\sum_{\mathrm{i}=1}^{\mathrm{s}} \mathrm{n}_{\mathrm{i}}
$$

(Krebs, 1989), onde $n_{i}$ é o número de citações por espécie (considera-se apenas uma citação por espécie por informante, mesmo que um informante tenha citado uma espécie várias vezes, para vários usos) e $\mathrm{N}$ é o número total de citações, conforme foi sugerido por Begossi (1996). Para estes cálculos, foi usado o programa Krebs para Windows (1997). Foi aplicado o teste $\mathrm{t}$ para se testar diferenças entre pares de índices de diversidade (Zar 1996).

Para se verificar a suficiência amostral, foram feitas as curvas de rarefação, através do programa Krebs para Ms-DOS (Krebs 1989).

\section{Resultados e discussão}

A área estudada caracteriza-se por uma situação sócio-econômica em transformação; o confronto entre modos de pensar e agir tradicionais e novas idéias e costumes trazidos com o contato intensificado nas décadas recentes com a sociedade nacional, reflete-se também nas questões ligadas à saúde e à doença. Hoje em dia, a população local conta com facilidades médicas, como um Centro de Saúde e um Hos- 
pital Municipal, e, embora o emprego das plantas com fins terapêuticos ainda seja parte importante do cotidiano da maioria, existem outras opções disponíveis de tratamento.

As espécies foram coletadas de ambientes diversos, como quintais, roças, áreas com vegetação em sucessão secundária, cerrados e áreas periodicamente alagadas. Pertencem a 73 famílias e 228 espécies; as famílias mais bem representadas foram Euphorbiaceae (17 espécies), Asteraceae (15 espécies), Caesalpiniaceae (13 espécies) e Lamiaceae (12 espécies), Fabaceae e Poaceae (9 espécies) e Solanaceae (8 espécies). Cerca de $56 \%$ das espécies crescem espontaneamente em ambientes naturais ou antropicamente modificados, enquanto $41 \%$ são cultivadas localmente; o restante é adquirido por compra. O Apêndice 1 apresenta as espécies citadas, suas características botânicas e ecológicas. O maior número de espécies foi indicado para doenças do aparelho digestivo e aparelho respiratório (Fig. 2). Resultados semelhantes têm sido registrados para outros locais, tanto no Brasil (Silva-Almeida \& Amorozo 1998; Hanazaki et al 1996; Amorozo \& Gély 1988), quanto em outras partes da América Latina (Bennett \& Prance 2000; Trotter II 1981). Em seguida, vêm as doenças do aparelho genito-urinário e lesões e outras consequências de causas externas. A maior parte das espécies tem mais de uma indi- cação terapêutica. Todas as partes vegetais foram indicadas para o preparo de remédios; as mais utilizadas foram as folhas (incluindo ramos e brotos - 126 espécies), raízes (41), cascas (38) e planta inteira (31). É de se notar que o uso de raízes e cascas foi mais comum para plantas do cerrado. Frutos (25 espécies), sementes (16), flores (15) e latex ou seiva (11) foram indicados menor número de vezes. Diferentes partes da mesma espécie podem ser empregadas de diferentes modos, para a mesma afecção, ou para diferentes afecções. O modo de administração mais comum foi por via oral (161 espécies), principalmente sob forma de chás (infusão ou decocto - 124 espécies), seguido por maceração em água ou cachaça (25) e xarope (25); 83 espécies foram indicadas para banhos ou outro tipo de aplicação externa.

Os valores dos índices de diversidade encontrados comparam-se aos mais altos registrados para plantas medicinais em outras regiões tropicais (Begossi 1996; Figueiredo et al. 1993). A Tab. 1 apresenta os resultados para o presente trabalho e para um estudo feito em área de floresta pluvial tropical em Barcarena, no Pará (Amorozo \& Gély 1988, Amorozo \& Gély, dados não publicados e Amorozo 1993), para o total das espécies, para as espécies espontâneas e as cultivadas. Tanto a riqueza, quanto os valores dos índices de diversidade, em to-

Tabela 1. Índices de diversidade de plantas medicinais

\begin{tabular}{|c|c|c|c|c|c|c|}
\hline \multirow[t]{2}{*}{ Local } & \multirow[t]{2}{*}{$\begin{array}{c}\text { Status } \\
\text { ecológico }\end{array}$} & \multirow[t]{2}{*}{ Riqueza } & \multicolumn{2}{|c|}{$\begin{array}{l}\text { Índice de Shannon- } \\
\text { Wiener }\end{array}$} & \multirow[t]{2}{*}{ Equitabilidade } & \multirow[t]{2}{*}{$\begin{array}{c}\text { Total } \\
\text { de Citações }\end{array}$} \\
\hline & & & base 10 & base e & & \\
\hline S.Antonio do & espontâneas ${ }^{1}$ & 127 & 1,95 & 4,48 & 0,93 & 528 \\
\hline Leverger, & cultivadas & 94 & 1,84 & 4,24 & 0,93 & 380 \\
\hline MT & total $^{2}$ & 228 & 2,21 & 5,09 & 0,94 & 938 \\
\hline Barcarena, & espontâneas ${ }^{1}$ & 142 & 2,03 & 4,67 & 0,94 & 327 \\
\hline \multirow[t]{2}{*}{ PA } & cultivadas & 114 & 1,93 & 4,45 & 0,94 & 432 \\
\hline & total $^{2}$ & 259 & 2,28 & 5,24 & 0,94 & 764 \\
\hline
\end{tabular}

${ }^{1}$ inclui espécies espontâneas que também são cultivadas na área.

2 inclui espécies obtidas de outras formas. 
dos os casos, são maiores para Barcarena, do que para Santo Antonio. Também, para ambas as áreas, diversidade e riqueza são maiores em relação às plantas espontâneas - aí incluídas tanto as silvestres como as invasoras - do que em relação às cultivadas. A Tab. 2 apresenta os resultados do teste $t$ para diferenças entre pares de índices de diversidade (Zar 1996). Todas as comparações foram estatisticamente significantes.

A equitabilidade na amostra total é alta em ambos os locais; Santo Antonio apresenta um valor um pouco menor, com relação às espécies espontâneas e cultivadas. Equitabilidade alta, como é o caso, mostra que o conhecimento sobre uso terapêutico de plantas tem distribuição relativamente uniforme entre os indivíduos da amostra estudada.

Comparando-se as curvas de rarefação para número de citações (Fig. 3), verifica-se que a curva para Barcarena tem inclinação mais acentuada do que a de Santo Antonio, apresentando maior número de espécies por unidade de esforço amostral; neste caso, é provável que um aumento no tamanho da amostra acarretasse ainda aumento na riqueza observada.

Um dos fatores que influencia o conhecimento e uso de plantas medicinais é a disponibilidade de espécies a serem utilizadas. A maior diversidade de espécies de Barcarena em relação a Santo Antonio, bem como os resultados das curvas de rarefação, estão congruentes com a riqueza florística dos ambientes ocupados pelas populações estudadas, já que, de modo geral, a floresta amazônica é mais rica em espécies do que o cerrado.

A diversidade de espécies espontâneas utilizadas medicinalmente reflete até certo ponto a riqueza florística local; a diversidade significativamente maior de plantas espontâneas em relação às cultivadas está ligada à disponibilidade de habitats (florestas, cerrado, vegetação secundária) e ao fato de que estas populações exploram efetivamente estes ambientes na procura de plantas medicinais. Em Santo Antonio, mais de um terço das espécies espontâneas é nativo do cerrado.

Quanto à diversidade de espécies cultivadas, que geralmente são exóticas, depende principalmente de aportes externos. Em ambas as áreas, pouco mais de $40 \%$ das espécies medicinais são cultivadas localmente. Bennett \& Prance (2000) chamam a atenção para a importância das espécies introduzidas na farmacopéia vegetal de povos índigenas e mestiços do Norte da América do Sul. Muitas destas plantas, segundo estes autores, foram introduzidas na época da conquista européia, para fins alimentares e ornamentais, e seu uso acabou sendo estendido à cura de enfermidades. Gabriel Soares de Sousa, em 1587 (Sousa 1974), relata a aclimatação de muitas plantas trazidas de além-mar para a Bahia, como banana, canade-açúcar, romã, cítricos, gengibre, hortelã, couve, alface, cebola, alho e poejos, entre outras, que são atualmente usadas de forma extensiva como medicinais. Mais especificamente para a região de Mato Grosso, Riedel, botânico da Expedição Langsdorff, que percorreu a região entre 1827 e 1828 , assinala o uso medicinal de algumas plantas cultivadas, como o fumo, a goiaba, a maravilha e o urucu (Saddi 1993).

A composição de uma farmacopéia popular é, pois, um processo dinâmico, durante o qual podem ocorrer tanto aquisições como perdas. Nas situações onde o contato com a sociedade em geral ou com migrantes se intensifica, é possível que aumentem as oportunidades, tanto de entrada de novas espécies, antes inexistentes na área, que são testadas pela população local, quanto de novos usos para espécies já existentes. Â medida que estas plantas e informações recém-introduzidas vão se disseminando, também o número de pessoas que as usam poderá aumentar. Desta forma, a riqueza e diversidade de espécies usadas medicinalmente podem aumentar, pelo menos durante um período. As comunidades de agricultores estudadas em Mato Grosso estão submetidas a um processo de mudança e exposição a fatores externos bastante acelerado; verificou-se, neste caso, a entrada de 


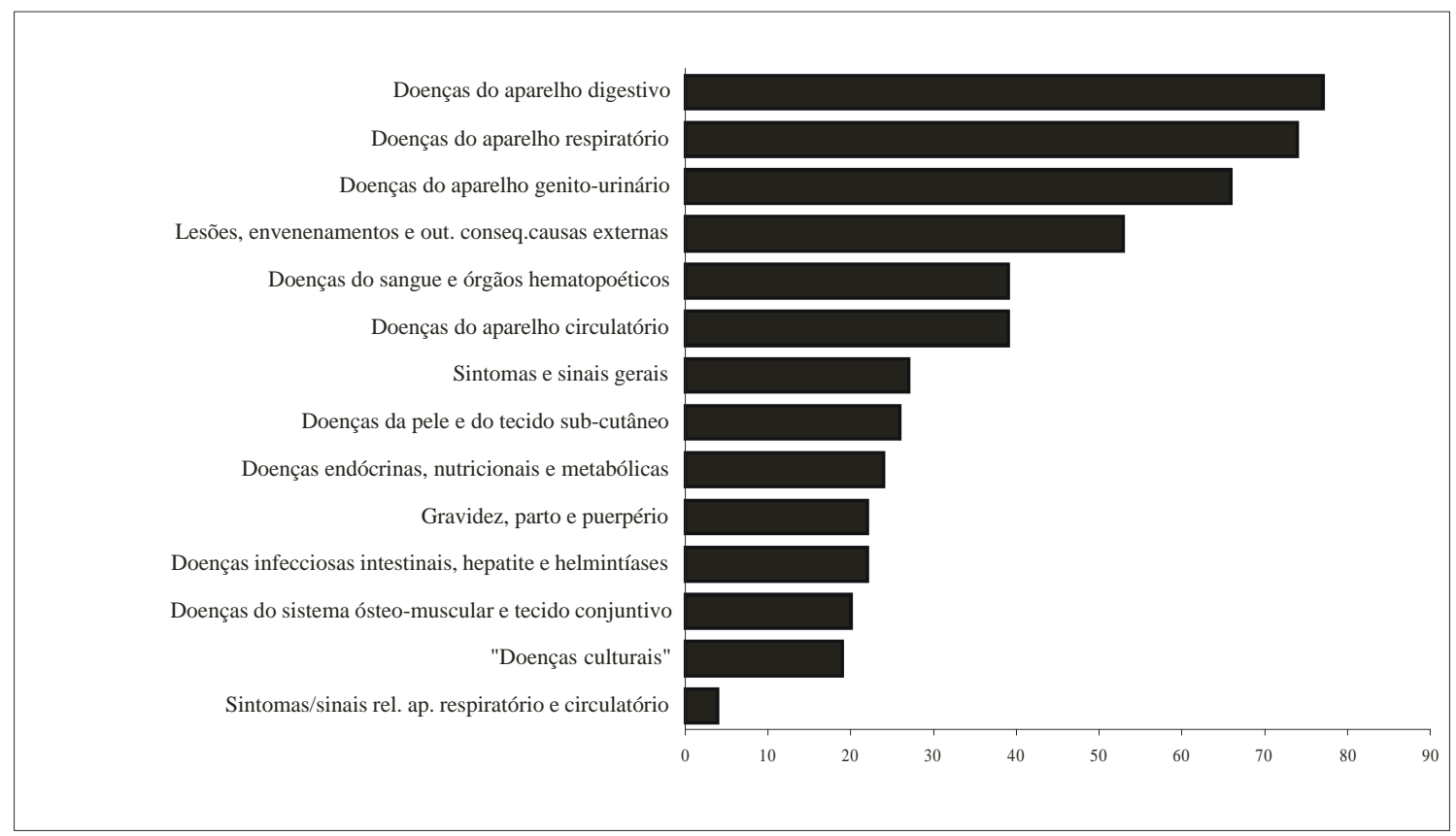

Figura 2. Número de espécies indicada por grupos de afecções.

novas informações com relação à utilização de plantas para fins medicinais através de veículos de comunicação de massa, principalmente a televisão (por exemplo, o uso da pata-de-vaca (Bauhinia spp.) contra diabetes, relatado por um informante; a utilização da resina da ameciqueira (Protium heptaphyllum (Aubl.) March) como medicinal antes usavam apenas casca, folha e brotos - relatada por outro) e contato com pessoas de fora das comunidades (por exemplo, o uso da coroa-decristo (Euphorbia milii des Moulins), uma espécie ornamental, para problemas do coração).

Por outro lado, vários fatores contribuem para que haja perda de espécies de valor terapêutico e de informações sobre elas; a alteração antrópica, ocasionada por mudanças nos padrões de uso local dos ambientes naturais, onde crescem muitas das espécies medicinais, irá, a médio prazo, acarretar uma diminuição na disponibilidade e no uso de plantas nativas e espontâneas para estes fins. Estas mudanças começam a ser sentidas em Santo Antonio, onde os entrevistados afirmavam que muitas das espécies nativas de cerrado estavam se tornando difíceis de encontrar, seja pela destruição dos habitats, para formação de pastagens ou uso urbano, seja pela sua inacessibilidade, devido à apropriação e cercamento das terras por indiví-

Tabela 2. Teste $\mathrm{t}$ para diferenças entre os índices de diversidade segundo localidade e status ecológico. SAL=Santo Antonio do Leverger, MT; Bar=Barcarena, PA; C=cultivada; E=espontânea

\begin{tabular}{cccccc}
\hline $\begin{array}{c}\text { Local e status } \\
\text { ecológico }\end{array}$ & $\begin{array}{c}\text { Bar x SAL } \\
\text { (total) }\end{array}$ & $\begin{array}{c}\text { Ex C } \\
(\mathrm{SAL})\end{array}$ & $\begin{array}{c}\mathrm{Ex} \mathrm{C} \\
(\mathrm{Bar})\end{array}$ & $\mathrm{C} \times \mathrm{C}$ & $\mathrm{E} \times \mathrm{E}$ \\
$\mathrm{t}$ & $4,033^{* *}$ & $4,909 * *$ & $4,047 * *$ & $4,267 * *$ & $3,387 * *$ \\
g.l. & 1627 & 859 & 665 & 793 & 705 \\
\hline
\end{tabular}

$* * \mathrm{p}<0,001$ 


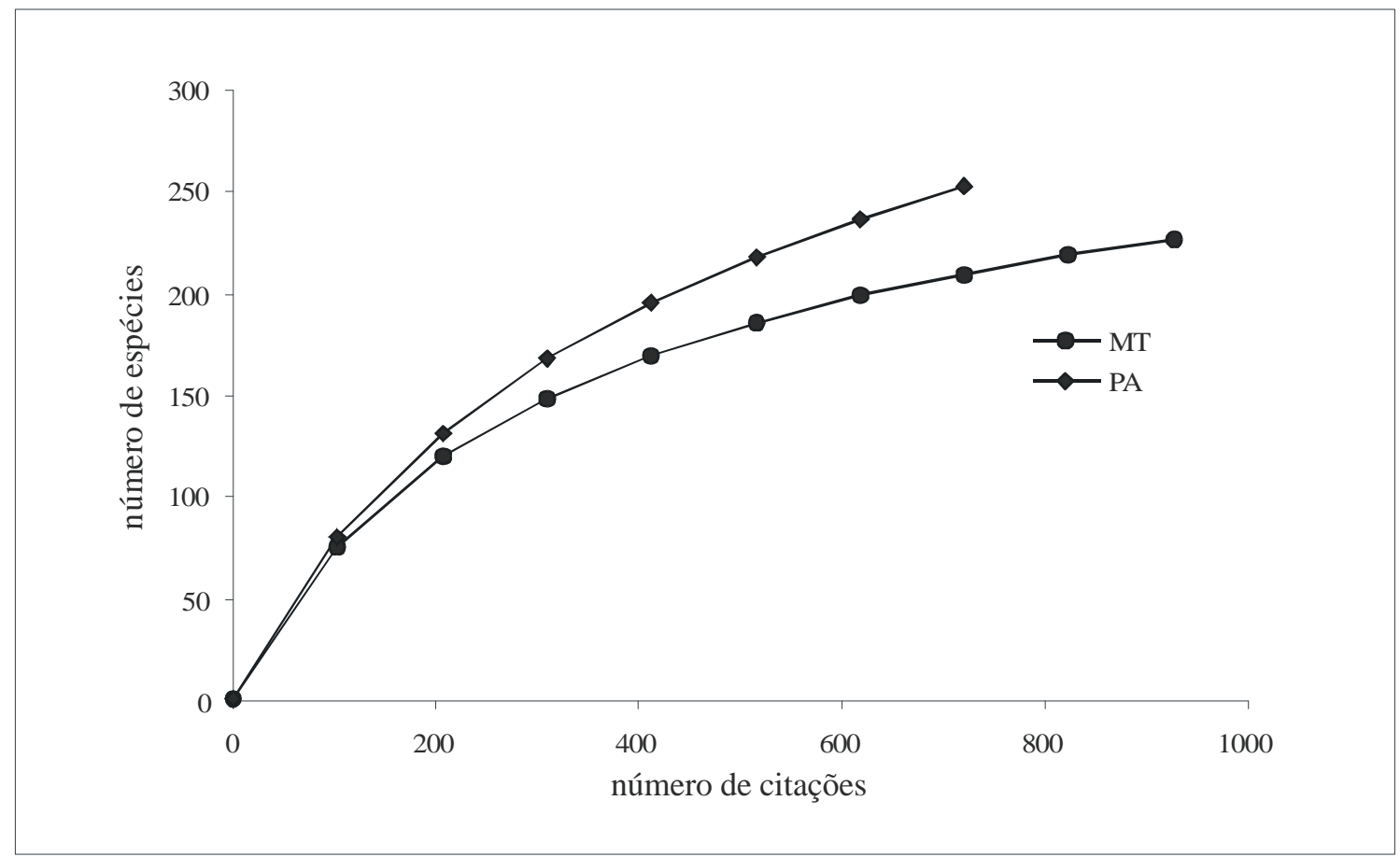

Figura 3. Número de citações X número de espécies em duas áreas estudadas.

duos estranhos às comunidades. Também, a "modernização" traz consigo novas opções de cuidados com a sáude, e uma certa desvalorização da cultura local, à qual os jovens são o grupo mais sensível, reforçando a tendência à perda ou abandono das práticas tradicionais.

Tal processo encontra-se em estado já avançado em áreas inseridas há mais tempo na economia nacional, como é o caso do Estado de São Paulo. Em um estudo etnobotânico de um bairro rural no município de Conchas, SP, Dias (1999) registrou 80 espécies com usos medicinais, e índices de diversidade de 1,76 e 4,05 (ShannonWiener bases 10 e e, respectivamente), resultados menores, portanto, do que os aqui descritos. Ademais, a autora nota que cerca de $64 \%$ das espécies provêm de cultivo. Em outro distrito, próximo a Rio Claro, SP, Silva-Almeida \& Amorozo (1998) encontraram 70 espécies de plantas com usos medicinais, ressaltando que a maioria delas era cultivada, sendo quase $79 \%$ obtidas de quintais e jardins (incluindo-se aí tanto as cultivadas como as toleradas); neste último local, os ambientes naturais haviam cedido espaço, em sua maior parte, para pastagens e terrenos de cultivo.

Sugere-se, desta forma, que quando comunidades tradicionais se tornam mais expostas à sociedade nacional, o conhecimento e o uso de plantas medicinais podem sofrer inicialmente um acréscimo, com o aumento das oportunidades de contato com espécies exóticas e informações sobre elas. Mas, à medida que este processo vai se aprofundando, ocasionando modificações nas formas de apropriação e uso da terra, com a substituição de ambientes naturais por artificiais, à medida que novos valores se sobrepõem aos antigos, e aumenta o acesso a cuidados institucionalizados com a saúde, a tendência é que a diversidade de plantas utilizadas com fins terapêuticos se torne restrita às espécies cultivadas e às invasoras cosmopolitas. 


\section{Agradecimentos}

À FAPESP, pelo auxílio à pesquisa processo $n^{\mathrm{o}}$ 1996/8127-2. Ao povo de Santo Antonio do Leverger, MT, pela acolhida, pela colaboração irrestrita e pela amizade. Ao Miguel Petrere Jr. e Leila Cunha de Moura, pela leitura crítica do manuscrito e sugestões; a dois revisores anônimos, pelas correções e recomendações.

\section{Referências bibliográficas}

Alvarenga, S.M.; Brasil, A.E.; Pinheiro, R. \& Kux, H.J.H. 1984. Estudo geomorfológico aplicado à bacia do rio Paraguai e pantanais matogrossenses. Boletim Técnico 1, Série Geomorfologia. Projeto RADAM-BRASIL - Salvador, BA, $183 \mathrm{p}$.

Amorozo, M.C.M. \& Gély, A.L. 1988. Uso de plantas medicinais por caboclos do baixo Amazonas, Barcarena, PA, Brasil. Boletim do Museu Paraense Emílio Goeldi, Série Botânica 4(1):47-131.

Amorozo, M.C.M. 1993. Algumas notas adicionais sobre o emprego de plantas e outros produtos com fins terapêuticos pela população cabocla do município de Barcarena, PA, Brasil. Boletim do Museu Paraense Emílio Goeldi, Série Botânica 9(2):249-266.

Begossi, A. 1996. Use of ecological methods in ethnobotany: diversity indices. Economic Botany 50(3):280-289.

Bennett, B.C. \& Prance, G.T. 2000. Introduced plants in the indigenous pharmacopoeia of Northern South America. Economic Botany 54 (1):90-102

Bernard, H.R. 1988. Research methods in cultural anthropology. Newbury Park, CA: Sage Publ., 520 p.

Brasil, Ministério das Minas e Energia, 1982. Folha SD-21 Cuiabá. Levantamento de recursos naturais. Secretaria Geral, Projeto RADAM-BRASIL. RJ, 544 p.

Brunelli, G. 1987. Des esprits aux microbes - santé et societé en transformation chez les Zoró de l'Amazonie brésilienne. Tese de Mestrado, Département d'Anthropologie, Faculté des Arts et des Sciences, Université de Montréal, 471 p.

Dias, M.C. 1999. Plantas medicinais utilizadas no Distrito de Juquiratiba - Município de Conchas - SP.
Dissertação de Mestrado em Agronomia - área de concentração Horticultura. Faculdade de Ciências Agronômicas da UNESP, Campus de Botucatu, 82p.

Farnsworth, N.R. 1988. Screening plants for new medicines. In: Wilson, E.O. (ed) Biodiversity .Washington DC: Nac. Acad. Press, 521p.

Figueiredo, G.M.; Leitão-Filho, H.F. \& Begossi, A. 1993. Ethnobotany of atlantic forest coastal communities: diversity of plant uses in Gamboa (Itacuruçá Island, Brazil). Human Ecology 21(4):419-430

Hanazaki, N.; Leitão-Filho, H.F. \& Begossi, A. 1996. Uso de recursos na mata atlântica: o caso do Pontal do Almada (Ubatuba, Brasil). Interciencia 21 (6):268-276

Krebs, C.J. 1989. Ecological methodology. New York: Harper \& Row, Publ., 654 p.

Organização Mundial da Saúde, 2000. Classificação Estatística Internacional de Doenças e Problemas Relacionados à Saúde. 10 $0^{\mathrm{a}}$ revisão, São Paulo: Editora da Universidade de São Paulo, Vol. 1, 1191 p.

Phillips, O. \& Gentry, A. H. 1993a. The useful plants of Tambopata, Peru: I. Statistical hypotheses tests with a new quantitative technique. Economic Botany 47(1):15-32.

Phillips, O. \& Gentry, A. H. 1993b. The useful plants of Tambopata, Peru: II. Aditional hypothesis testing in quantitative ethnobotany. Economic Botany 47(1):33-43

Prance, G.T; Balée, W.; Boom, B.M. \& Carneiro, R.L. 1987. Quantitative ethnobotany and the case for conservation in Amazonia. Conservation Biology 1(4):296-310.

Saddi, N. 1993. Acerca das observações de Riedel, botânico da Expedição Langsdorff, em Mato Grosso (Brasil), no Manuscrito de D'Alincourt. In: Costa, M.F.G. (org.) Percorrendo manuscritos entre Langsdorff e D'Alincourt. Cuiabá, Ed. Universitária, p 36-49

Silva-Almeida, M.F. \& Amorozo, M.C.M., 1998. Medicina popular no Distrito de Ferraz, Município de Rio Claro, Estado de São Paulo. Brazilian Journal of Ecology 2:36-46.

Sousa, G.S. 1974. Notícias do Brasil. SP, Brasiliensia Documenta, Vol. VII, $486 \mathrm{p}$

Trotter II, R.T. 1981. Folk remedies as indicators of common illnesses: examples from the United States - Mexico border. Journal of Ethnopharmacology $4: 207-221$

Zar, J.H. 1996. Biostatistical analysis. New Jersey: Prentice Hall, $3^{\mathrm{d}}$ ed., $662 \mathrm{p}+$ apêndices 
Apêndice 1. Plantas com aplicação terapêutica

Legenda: Ref=número de herbário ou de coleta $(\mathrm{CA}=$ M.C.Amorozo; $\mathrm{MC}=\mathrm{M}$.Carvalho; $\mathrm{RS}=\mathrm{R}$. Silva $)$; il=identificada no local; NI=número de informantes citando a planta; Hab=Hábito: avo=árvore; arb=arbusto; sab=subarbusto; erv=herbácea; epf=epífita; hepf=hemiepífita; trp=trepadeira; est=estipe; hpar=hemiparasita; *=comprada; c=cultivada; e=espontânea

\begin{tabular}{|c|c|c|c|c|c|c|}
\hline Nome científico & Nome vulgar & Ref & NI & Hab & Habitat / local de coleta & $\mathbf{C} / \mathbf{E}$ \\
\hline \multicolumn{7}{|l|}{ Polypodiaceae } \\
\hline Polypodium leucatomus Poir. & $\begin{array}{l}\text { rabo-de-macaco, rabo-de- } \\
\text { caxinguelê, lã-de-carneiro }\end{array}$ & 30770 & 3 & epf & beira de rio & $\mathrm{e}$ \\
\hline \multicolumn{7}{|l|}{ Acanthaceae } \\
\hline Justicia cf. pectoralis Jacq. & anador & CA277 & 5 & erv & quintal & $\mathrm{c}$ \\
\hline Justicia sp. & olho-grande & 30806 & 2 & arb & quintal & $\mathrm{c}$ \\
\hline \multicolumn{7}{|l|}{ Alismataceae } \\
\hline Echinodorus sp. & chapéu-de-couro & CA310 & 4 & erv & brejo ou local alagado & $\mathrm{e}$ \\
\hline \multicolumn{7}{|l|}{ Alliaceae } \\
\hline Allium cepa $\mathrm{L}$. & cebola & - & 3 & erv & $*$ & \\
\hline Allium sativum $\mathrm{L}$. & alho & il & 7 & erv & roça na beira de rio & $\mathrm{c}$ \\
\hline \multicolumn{7}{|l|}{ Amaranthaceae } \\
\hline Alternanthera brasiliana (L.) Kuntze & terramicina & 30790 & 5 & erv & quintal & $\mathrm{c}$ \\
\hline Amaranthus cf. lividus L. & caruru-de-porco & 30787 & 1 & erv & terreno baldio & $\mathrm{e}$ \\
\hline Gomphrena celosioides Mart. & perpetinha & 30789 & 1 & erv & ambiente perturbado em beiro de rio & $\mathrm{e}$ \\
\hline \multicolumn{7}{|l|}{ Amaryllidaceae } \\
\hline Crinum procerum Carey & lírio & 30788 & 1 & erv & sítio & $\mathrm{c}$ \\
\hline \multicolumn{7}{|l|}{ Anacardiaceae } \\
\hline Anacardium humile St.Hil. & cajuzinho, cajueiro-do-mato & CA16 & 3 & $\mathrm{sab}$ & cerrado & $\mathrm{e}$ \\
\hline Anacardium occidentale $\mathrm{L}$. & caju-branco & il & 2 & avo & quintal & $\mathrm{c}$ \\
\hline Astronium fraxinifolium Schott & gonçaleiro & CA297 & 1 & avo & beira de estrada & $\mathrm{e}$ \\
\hline Mangifera indica $\mathrm{L}$. & manga, mangueira & il & 7 & avo & chácara, quintal & $\mathrm{c}$ \\
\hline Myracrodruon urundeuva Fr.All. & aroeira & CA261 & 10 & avo & cerrado & $\mathrm{e}$ \\
\hline \multicolumn{7}{|l|}{ Apiaceae } \\
\hline Pimpinella sp. & erva-doce & - & 14 & erv & $*$ & \\
\hline \multicolumn{7}{|l|}{ Apocynaceae } \\
\hline Catharanthus roseus (L.) Don. & boa-noite & 30791 & 2 & erv & jardim & $\mathrm{c}$ \\
\hline Hancornia speciosa Gomez & mangabeira-mansa & 30805 & 4 & avo & cerrado & e \\
\hline Himatanthus obovatus (Muell.Arg.) Woods & angélica, tiborna & CA39 & 7 & avo & cerrado & $\mathrm{e}$ \\
\hline Macrosiphonia longiflora (Desf.) Muell. Arg. & velame, velame-do-campo & 30807 & 6 & sab & cerrado & $\mathrm{e}$ \\
\hline \multicolumn{7}{|l|}{ Araceae } \\
\hline cf. Monstera sp. & costela-de-adão & CA390 & 1 & hepf & quintal & $\mathrm{c}$ \\
\hline Philodendron cf. tripartitum Schott & cipó-de-caboclo & CA362 & 1 & $\operatorname{trp}$ & quintal & $\mathrm{c}$ \\
\hline \multicolumn{7}{|l|}{ Arecaceae } \\
\hline Acrocomia aculeata (Jacq.) Lodd. ex Mart. & bocaiúva, bocaiuveira & il & 2 & est & cerrado & $\mathrm{e}$ \\
\hline Allagoptera cf. leucocalix (Drude) O. Kuntze & oriri, ariri & MC41 & 1 & est & cerrado & $\mathrm{e}$ \\
\hline Cocos nucifera $\mathrm{L}$. & coco, coco-da-bahia & il & 5 & est & quintal & $\mathrm{c}$ \\
\hline \multicolumn{7}{|l|}{ Asteraceae } \\
\hline \multirow[t]{2}{*}{ Acanthospermum hispidum DC. } & $\begin{array}{l}\text { chifre-de-garrote, cabeça- } \\
\text { de-garrotinho }\end{array}$ & 30707 & 4 & erv & terreiro & $\mathrm{e}$ \\
\hline & & 30709 & & & & \\
\hline Ageratum conyzoides $\mathrm{L}$. & mentraste & 30682 & 2 & erv & cerrado & $\mathrm{e}$ \\
\hline \multirow[t]{2}{*}{ Artemisia absinthium L. } & losna, novônica-macho & CA432 & 10 & erv & quintal & $\mathrm{c}$ \\
\hline & & CA313 & & & & \\
\hline Artemisia verlotorum Lamotte & artemije, artemijo & 30664 & 4 & erv & quintal & $\mathrm{c}$ \\
\hline \multirow[t]{2}{*}{ Bidens pilosa $\mathrm{L}$. } & picão-roxo, picão-preto & 30838 & 3 & erv & quintal & $\mathrm{e}$ \\
\hline & & 30839 & & & & \\
\hline Centratherum punctatum Cass. & sem nome & 30708 & 1 & erv & quintal & $\mathrm{c}$ \\
\hline Chrysanthemum sp. & camomila-fêmea & 30676 & 1 & erv & quintal & $\mathrm{c}$ \\
\hline Dendrathema grandifolia (Ram.) Tzv. & $\begin{array}{l}\text { camomila-branca, camomila- } \\
\text { amarela }\end{array}$ & 30677 & 1 & erv & quintal & $\mathrm{c}$ \\
\hline Egletes viscosa Less. & marcela & 30683 & 4 & erv & beira de rio & $\mathrm{e}$ \\
\hline Lactuca sativa $\mathrm{L}$. & alface & - & 1 & erv & $*$ & \\
\hline Matricaria sp. & camomila, camomila-rauliveira & 30833 & 4 & erv & quintal & $\mathrm{c}$ \\
\hline Orthopappus angustifolius (SW.) Gleason & sansoaiá & 30706 & 1 & erv & cerrado & $\mathrm{e}$ \\
\hline \multirow[t]{3}{*}{ Porophyllum ruderale (Jacq.) Cass. } & picão, picão-branco & 30665 & 9 & erv & beira de roça, quintal & $\mathrm{e}$ \\
\hline & & 30814 & & & & \\
\hline & & 30836 & & & & \\
\hline Vernonia brasiliana Druce & assa-peixe & 30705 & 8 & arb & veg. sec. & $\mathrm{e}$ \\
\hline Vernonia condensata Baker & boldo-de-árvore, bordo, & 30830 & 8 & avo & quintal & $\mathrm{c}$ \\
\hline
\end{tabular}


Apêndice 1. (continuação).

\begin{tabular}{|c|c|c|c|c|c|c|}
\hline Nome científico & Nome vulgar & Ref & NI & Hab & Habitat / local de coleta & $\mathbf{C} / \mathbf{E}$ \\
\hline \multicolumn{7}{|l|}{ Asteraceae (cont.) } \\
\hline & $\begin{array}{l}\text { estomalina, cancerosa, canforona, } \\
\text { caferana, cura-tudo }\end{array}$ & 30840 & & & & \\
\hline \multicolumn{7}{|l|}{ Bignoniaceae } \\
\hline Anemopaegma arvense (Vell.) Stelf. & alecrim, alecrim-do-campo & 30750 & 3 & erv & cerrado & e \\
\hline Crescentia cujete $\mathrm{L}$. & cabaça-do-ar & CA409 & 2 & avo & quintal & $\mathrm{c}$ \\
\hline Cybistax antisyphilitica (Mart.) Mart. & pé-de-anta & MC5 & 6 & arb & cerrado & $\mathrm{e}$ \\
\hline Tabebuia aurea (Manso) B. et $\mathrm{H}$. & paratudo, pratudinho & 30755 & 10 & avo & cerrado & $\mathrm{e}$ \\
\hline Tabebuia impetiginosa (Mart. ex DC) Standl. & piúva-roxa, piúva-preta & $\begin{array}{l}30749 \\
30754\end{array}$ & 3 & avo & baixada, beira do rio & $\mathrm{e}$ \\
\hline $\begin{array}{l}\text { Tabebuia ochracea (Cham.) Standl. } \\
\text { Bixaceae }\end{array}$ & piúva amarela, ipê & CA245 & 2 & avo & cerrado & $\mathrm{e}$ \\
\hline $\begin{array}{l}\text { Bixa orellana } \mathrm{L} . \\
\text { Boraginaceae }\end{array}$ & urucum & il & 6 & avo & quintal & $\mathrm{c}$ \\
\hline Cordia insignis Cham. & calção-de-velho, santa-cruz & 30747 & 5 & arb & cerrado & $\mathrm{e}$ \\
\hline $\begin{array}{l}\text { Heliotropium indicum } \mathrm{L} \text {. } \\
\text { Brassicaceae }\end{array}$ & crista-de-galo & 30751 & 1 & erv & cerrado, lugares úmidos & $\mathrm{e}$ \\
\hline \multicolumn{7}{|l|}{ Bromeliaceae } \\
\hline Ananas comosus (L.) Merr. & abacaxi & il & 1 & erv & roça & $\mathrm{c}$ \\
\hline Bromelia balansae $\mathrm{Mez}$ & gravatá & 30745 & 4 & erv & cerrado & $\mathrm{e}$ \\
\hline \multicolumn{7}{|l|}{ Burseraceae } \\
\hline \multicolumn{6}{|l|}{ Cactaceae } & $\mathrm{e}$ \\
\hline \multicolumn{7}{|l|}{ Caesalpiniaceae } \\
\hline Bauhinia acuruana Moric. & pata-de-vaca & 30847 & 1 & avo & cerrado & $\mathrm{e}$ \\
\hline Bauhinia cf ungulata L.S. Lat & pata-de-vaca & CA327 & 1 & avo & quintal & $\mathrm{e}$ \\
\hline Bauhinia glabra Jacq. & cipó-tripa-de-galinha & 30774 & 3 & $\operatorname{trp}$ & cerrado & $\mathrm{e}$ \\
\hline Bauhinia mollis (Bong.) D.Dietr. & pata-de-vaca & CA346 & 1 & avo & urbano & $\mathrm{c}$ \\
\hline Bauhinia rufa (Bong.) Steud. & $\begin{array}{l}\text { pata-de-vaca, pé-de-vaca, pé-de- } \\
\text { boi }\end{array}$ & 30851 & 2 & avo & cerrado & $\mathrm{e}$ \\
\hline Caesalpinia ferrea Mart. & jucá & 30765 & 2 & avo & quintal & $\mathrm{c}$ \\
\hline Caesalpinia pulcherrima (L.) Sw. & chaga & 30764 & 1 & avo & urbano & $\mathrm{c}$ \\
\hline Chamaecrista desvauxii (Collad.) Killip & sene & 30766 & 7 & sab & cerrado & $\mathrm{e}$ \\
\hline $\begin{array}{l}\text { Hymenaea courbaril L. var. stilbocarpa } \\
\text { (Hayne) Lee et Lang. }\end{array}$ & jatobá-mirim & CA289 & 8 & avo & cerrado & $\mathrm{e}$ \\
\hline Hymenaea stigonocarpa Mart. ex Hayne & jatobá-açu & 30773 & 1 & avo & cerrado, vargem & $\mathrm{e}$ \\
\hline Senna alata (L.) Roxb. & mata-passo, mata-pasto & RS5 & 6 & $\mathrm{sab}$ & vargem, cerrado & $\mathrm{e}$ \\
\hline Senna occidentalis (L.) Link & fedegoso & 30849 & 11 & $\mathrm{sab}$ & beira de estrada & $\mathrm{e}$ \\
\hline $\begin{array}{l}\text { Tamarindus indica } \mathrm{L} \text {. } \\
\text { Capparaceae }\end{array}$ & tamarindo, tamarino & 30763 & 8 & avo & quintal & $\mathrm{c}$ \\
\hline Cleome aculeata DC. & quicendê, caçandé, cristian-guei & $\begin{array}{l}30759 \\
30762\end{array}$ & 3 & erv & terreno baldio & e \\
\hline $\begin{array}{l}\text { Cleome cf. spinosa } \mathrm{L} \text {. } \\
\text { Caprifoliaceae }\end{array}$ & tranca-rua & 30778 & 2 & arb & cerrado, vargem & $\mathrm{e}$ \\
\hline $\begin{array}{l}\text { Sambucus australis } \mathrm{C} \text {. et } \mathrm{S} \text {. } \\
\text { Caricaceae }\end{array}$ & sabugueiro & 30760 & 4 & arb & quintal & $\mathrm{c}$ \\
\hline Carica papaya $\mathrm{L}$ & mamão, mamão-macho & il & 9 & avo & roça, quintal & $\mathrm{c}$ \\
\hline Caryocaraceae & & & & & & \\
\hline $\begin{array}{l}\text { Caryocar brasiliense Camb. } \\
\text { Cecropiaceae }\end{array}$ & pequi & 30666 & 2 & avo & cerrado, vargem & $\mathrm{e}$ \\
\hline $\begin{array}{l}\text { Cecropia pachystachya Trec. } \\
\text { Celastraceae }\end{array}$ & embaúva & 30681 & 8 & avo & quintal & $\mathrm{e}$ \\
\hline $\begin{array}{l}\text { Maytenus cf. ilicifolia Mart. ex Reiss. } \\
\text { Chenopodiaceae }\end{array}$ & cancerosa & CA279 & 3 & arb & quintal & $\mathrm{c}$ \\
\hline $\begin{array}{l}\text { Chenopodium ambrosioides } \mathrm{L} \text {. } \\
\text { Commelinaceae }\end{array}$ & erva-de-santa-maria, mastruz & il & 22 & erv & quintal & $\mathrm{e}$ \\
\hline Callisia cf. repens L. & dinheiro-em-penca & CA363 & 1 & erv & quintal & $\mathrm{c}$ \\
\hline Commelina cf. nudiflora $\mathrm{L}$. & troperoba, copo-de-leite & 30819 & 1 & erv & cerrado, vargem & $\mathrm{e}$ \\
\hline Tradescantia zebrina Hort. ex Bosse & dinheiro-em-penca & CA364 & 1 & erv & quintal & $\mathrm{c}$ \\
\hline Convolvulaceae & & & & & & \\
\hline Evolvulus pterygophyllus Mart. & lã-de-carneiro & 30837 & 3 & erv & cerrado & $\mathrm{e}$ \\
\hline Ipomoea batatas (L.) Lam. & batata-doce & il & 4 & erv & roça & $\mathrm{c}$ \\
\hline
\end{tabular}


Apêndice 1. (continuação).

\begin{tabular}{|c|c|c|c|c|c|c|}
\hline Nome científico & Nome vulgar & Ref & NI & Hab & Habitat / local de coleta & $\mathbf{C} / \mathbf{E}$ \\
\hline \multicolumn{7}{|l|}{ Costaceae } \\
\hline Costus arabicus $\mathrm{L}$. & cana-de-macaco, cana-do-brejo & CA44 & 6 & erv & quintal & $\mathrm{e} / \mathrm{c}$ \\
\hline \multicolumn{7}{|l|}{ Crassulaceae } \\
\hline Kalanchoe pinnata Pers. & hei-de-vencer & 30776 & 1 & erv & quintal & $\mathrm{c}$ \\
\hline \multicolumn{7}{|l|}{ Cucurbitaceae } \\
\hline Citrullus lanatus (Thunb.) Mansf. & melancia & il & 1 & erv & roça & $\mathrm{c}$ \\
\hline Cucumis anguria $\mathrm{L}$. & maxixe & il & 1 & erv & roça & $\mathrm{c}$ \\
\hline Cucurbita $\mathrm{sp}$. & abóbora & il & 4 & erv & roça & $\mathrm{c}$ \\
\hline Momordica charantia $\mathrm{L}$. & são-caetano & 30820 & 12 & $\operatorname{trp}$ & lugares secos e úmidos & $\mathrm{e}$ \\
\hline Sechium edule Schwart & chuchu & - & 3 & $\operatorname{trp}$ & $*$ & \\
\hline \multicolumn{7}{|l|}{ Cyperaceae } \\
\hline Cyperus rotundus $\mathrm{L}$. & capim-trique-trique & 30679 & 1 & erv & calçada & $\mathrm{e}$ \\
\hline Rhynchospora nervosa Boeck. & pulsatilha & 30684 & 1 & erv & cerrado, vargem & $\mathrm{e}$ \\
\hline \multicolumn{7}{|l|}{ Dilleniaceae } \\
\hline Curatella americana $\mathrm{L}$. & lixeira & 30808 & 3 & avo & cerrado & $\mathrm{e}$ \\
\hline Davilla elliptica St.Hil. & lixinha & 30809 & 2 & arb & cerrado & $\mathrm{e}$ \\
\hline \multicolumn{7}{|l|}{ Droseraceae } \\
\hline Drosera aff. sessilifolia St.Hil. & ligeira & CA109 & 1 & erv & brejo & $\mathrm{e}$ \\
\hline \multicolumn{7}{|l|}{ Euphorbiaceae } \\
\hline Chamaesyce caecorum (Boiss.) Croizat & sete-sangria & 30803 & 5 & erv & cerrado & $\mathrm{e}$ \\
\hline Cnidosculus vitifolius Pohl. & cansançã & 30793 & 4 & sab & beira de estrada & $\mathrm{e}$ \\
\hline Croton bonplandianus Baillon & erva-de-rato & 30799 & 1 & erv & terreno baldio & $\mathrm{e}$ \\
\hline Euphorbia brasiliensis Lam. & quebra-pedra-legítimo & 30796 & 1 & erv & cerrado, beira de estrada & $\mathrm{e}$ \\
\hline Euphorbia milii des Moulins & coroa-de-cristo, não-me-toques & 30795 & 1 & sab & jardim em beira de muro & $\mathrm{c}$ \\
\hline \multirow[t]{2}{*}{ Euphorbia pilulifera $\mathrm{L}$. } & erva-de-santa-luzia, tranca-cu & 30786 & 2 & erv & quintal, calçada & $\mathrm{e}$ \\
\hline & & 30794 & & & & \\
\hline Euphorbia tymiifolia $\mathrm{L}$. & quebra-pedra & 30758 & 2 & erv & quintal, calçada & $\mathrm{e}$ \\
\hline Jatropha curcas $\mathrm{L}$. & pinhão-branco & 30792 & 5 & arb & jardim & $\mathrm{c}$ \\
\hline Jatropha elliptica (Pohl.) Muell. Arg. & raiz-de-lagarto, purga-de-lagarto & 30804 & 3 & erv & cerrado & $\mathrm{e}$ \\
\hline Jatropha gossypiifolia $\mathrm{L}$. & pinhão-roxo & 30797 & 5 & arb & quintal & $\mathrm{c}$ \\
\hline Manihot esculenta Crantz & mandioca & il & 5 & arb & roça & $\mathrm{c}$ \\
\hline Manihot sp. ? & bácimo-de-casa & CA374 & 2 & arb & quintal & $\mathrm{c}$ \\
\hline Phyllanthus niruri $\mathrm{L}$. & $\begin{array}{l}\text { quebra-pedra, quebra-pedra- } \\
\text { branco }\end{array}$ & CA80 & 1 & erv & roça & $\mathrm{e}$ \\
\hline Phyllanthus orbiculatus L.C.Rich. & $\begin{array}{l}\text { quebra-pedra legítimo, quebra- } \\
\text { pedra-roxo }\end{array}$ & 30811 & 3 & erv & cerrado & e \\
\hline Phyllanthus stipulatus (Raf.) Webster & $\begin{array}{l}\text { quebra-pedra, quebra-pedra- } \\
\text { branco }\end{array}$ & CA405 & 3 & erv & quintal & $\mathrm{e}$ \\
\hline \multirow[t]{2}{*}{ Ricinus communis L. } & mamona, óleo-de-rícino & 30798 & 5 & & terreiro & $\mathrm{e}$ \\
\hline & & 30800 & & avo & & \\
\hline Synadenium grantii Desmoul. ex Boiss. & cancerosa & CA270 & 1 & erv & quintal & $\mathrm{c}$ \\
\hline \multicolumn{7}{|l|}{ Fabaceae } \\
\hline Acosmium dasycarpum (Vog.) Yakovl. & quina-genciana, genciana & CA294 & 7 & arb & cerrado & $\mathrm{e}$ \\
\hline Bowdichia virgilioides H.B.K. & sucupira-roxa & 30854 & 2 & avo & cerrado & $\mathrm{e}$ \\
\hline Cajanus cajan (L.) Millsp. & feijão-andu & CA355 & 3 & arb & quintal & $\mathrm{c}$ \\
\hline Camptosema sp. ? & bácimo-do-cerrado & 30812 & 1 & erv & cerrado & $\mathrm{e}$ \\
\hline Desmodium cf incanum DC & carrapichinho & 30850 & 3 & erv & terreiro & $\mathrm{e}$ \\
\hline Dipteryx alata Vog. & cumbaru & 30813 & 2 & avo & cerrado & $\mathrm{e}$ \\
\hline Eriosema campestre Benth. & bácimo-da-chapada & 30852 & 1 & erv & cerrado & $\mathrm{e}$ \\
\hline Machaerium aculeatum Raddi & espinheira, espinheiro & CA269 & 8 & avo & brejo & $\mathrm{e}$ \\
\hline cf. Vatairea macrocarpa (Bth.) Ducke & gegelim & 30855 & 2 & avo & cerrado & $\mathrm{e}$ \\
\hline \multicolumn{7}{|l|}{ Flacourtiaceae } \\
\hline Casearia sylvestris Sw. & chá-de-frade & CA5 & 7 & $\mathrm{arb} /$ & cerrado & $\mathrm{e}$ \\
\hline & & & & avo & & \\
\hline \multicolumn{7}{|l|}{ Lamiaceae } \\
\hline Coleus sp. & boldo, bordo & il & 11 & arb & quintal & $\mathrm{c}$ \\
\hline Cunila microcephala Benth. & poejo & 30687 & 4 & erv & quintal & $\mathrm{c}$ \\
\hline Hyptis cf crenata Pohl. ex Benth. & hortelã & 30842 & 2 & erv & cerrado & $\mathrm{e}$ \\
\hline Hyptis goyazensis St.Hil. ex Benth. & hortelã-do-campo & 30674 & 1 & erv & cerrado & $\mathrm{e}$ \\
\hline Hyptis suaveolens Poir. & tapera-velha & 30688 & 8 & erv & beira de estrada & $\mathrm{e}$ \\
\hline cf. Lavandula spica Cav. & alfazema & 30673 & 1 & erv & quintal & $\mathrm{c}$ \\
\hline Leonotis nepetaefolia (L.) R.Brown & cordão-de-são-francisco & 30689 & 7 & erv & terreiro & $\mathrm{e}$ \\
\hline \multirow[t]{2}{*}{ Mentha arvensis L. var. piperascens Malinv. } & vick & 30671 & 2 & erv & quintal & $\mathrm{c}$ \\
\hline & & 30675 & & & & \\
\hline Mentha sp. & hortelãzinho & 30672 & 6 & erv. & quintal & $\mathrm{c}$ \\
\hline
\end{tabular}


Apêndice 1. (continuação).

\begin{tabular}{|c|c|c|c|c|c|c|}
\hline Nome científico & Nome vulgar & Ref & NI & Hab & Habitat / local de coleta & $\mathbf{C} / \mathbf{E}$ \\
\hline \multicolumn{7}{|l|}{ Lamiaceae (cont.) } \\
\hline Ocimum canum Sims. & alfavaquinha & 30844 & 3 & erv & quintal & $\mathrm{c}$ \\
\hline Ocimum gratissimum $\mathrm{L}$. & alfavaca & $\begin{array}{l}30822 \\
30823\end{array}$ & 7 & $\mathrm{sab}$ & quintal & $\mathrm{c}$ \\
\hline $\begin{array}{l}\text { Rosmarinus officinalis } \mathrm{L} \text {. } \\
\text { Lauraceae }\end{array}$ & alecrim-de-casa & il & 3 & sab & quintal & $\mathrm{c}$ \\
\hline $\begin{array}{l}\text { Persea americana Mill. } \\
\text { Liliaceae }\end{array}$ & abacate & il & 9 & avo & quintal & $\mathrm{c}$ \\
\hline $\begin{array}{l}\text { Herreria } \text { cf. salsaparilha Mart. } \\
\text { Loganiaceae }\end{array}$ & salsa & 30713 & 2 & erv & roça & $\mathrm{e}$ \\
\hline $\begin{array}{l}\text { Strychnos pseudoquina St.Hil. } \\
\text { Loranthaceae }\end{array}$ & quina & CA251 & 12 & avo & cerrado & $\mathrm{e}$ \\
\hline Psittacanthus calyculatus (DC.) C.Don & enxerto-de-passarinho & 30826 & 1 & hpar & quintal & $\mathrm{e}$ \\
\hline Psittacanthus cordatus Blume & enxerto-de-passarinho & 30757 & 1 & hpar & quintal & $\mathrm{e}$ \\
\hline $\begin{array}{l}\text { Struthanthus cf.. marginatus (Desr.) Blume } \\
\text { Lythraceae }\end{array}$ & enxerto-de-passarinho & 30827 & 1 & hpar & quintal - sobre tamarindo & $\mathrm{e}$ \\
\hline $\begin{array}{l}\text { Lafoensia } \text { cf. replicata } \text { Pohl. } \\
\text { Malpighiaceae }\end{array}$ & mangabeira-braba & MC48 & 16 & avo & cerrado & $\mathrm{e}$ \\
\hline Byrsonima verbascifolia (L.) Rich & birici & CA248 & 2 & avo & cerrado & $\mathrm{e}$ \\
\hline Camarea ericoides St.Hil. & arnica & 30668 & 12 & erv & cerrado & $\mathrm{e}$ \\
\hline Galphimia brasiliensis A. Juss. & mercúrio-crônico & 30828 & 1 & erv & campo sujo & $\mathrm{e}$ \\
\hline Heteropterys pannosa Griseb. & $\begin{array}{l}\text { nó-de-cachorro, raiz-de-santo- } \\
\text { antonio }\end{array}$ & MC3 & 6 & $\mathrm{sab}$ & cerrado & $\mathrm{e}$ \\
\hline Malvaceae & & & & & & \\
\hline Abelmoschus esculentus (L.) Moench & quiabo & il & 2 & erv & roça & $\mathrm{c}$ \\
\hline Abutilon sp .1 & marva, mave & $\begin{array}{l}30738 \\
30739\end{array}$ & 1 & erv & quintal & $\mathrm{e} / \mathrm{c}$ \\
\hline Abutilon sp. 2 & mave & 30737 & 1 & erv & quintal & $\mathrm{e} / \mathrm{c}$ \\
\hline Gossypium barbadense L. & algodão & 30735 & 10 & arb & quintal & $\mathrm{c}$ \\
\hline Hibiscus sabdariffa L. & quiabinho-de-angola & CA306 & 1 & arb & quintal & $\mathrm{c}$ \\
\hline Sida cordifolia $\mathrm{L}$. & malva-branca & $\begin{array}{l}30733 \\
30734\end{array}$ & 4 & erv & quintal & $\mathrm{c}$ \\
\hline Melastomataceae & & & & & & \\
\hline $\begin{array}{l}\text { Tibouchina } \text { cf. clavata (Pers.) Wurd. } \\
\text { Meliaceae }\end{array}$ & cibalena & CA445 & 1 & arb & quintal & $\mathrm{c}$ \\
\hline Cedrela $\mathrm{sp}$ & cedro & CA338 & 4 & avo & quintal & $\mathrm{c}$ \\
\hline $\begin{array}{l}\text { Melia azedarach L. } \\
\text { Mimosaceae }\end{array}$ & santa-bárbara & 30783 & 2 & avo & quintal & $\mathrm{c}$ \\
\hline Enterolobium contortisiliquum (Vell.) Morong. & ximbuva-branca & & 1 & avo & roça & $\mathrm{e}$ \\
\hline $\begin{array}{l}\text { Stryphnodendron adstringens (Mart.) Coville } \\
\text { Monimiaceae }\end{array}$ & barbatimão & CA264 & 11 & avo & cerrado & $\mathrm{e}$ \\
\hline Siparuna guianensis Aubl & negra-mina & 30740 & 10 & avo & cerrado & $\mathrm{e}$ \\
\hline Moraceae & & & & & & \\
\hline Artocarpus heterophyllus Lam. & jaca $\ldots$ & il & 1 & avo & quintal & $\mathrm{c}$ \\
\hline Brosimum gaudichaudii Tréc. & algodãozinho & CA441 & 5 & avo & cerrado & $\mathrm{e}$ \\
\hline Dorstenia asaroides Gardner & caiapiá & CA7 & 14 & erv & roça, cerrado & $\mathrm{e}$ \\
\hline Morus sp. & amora & il & 4 & avo & quintal & $\mathrm{c}$ \\
\hline $\begin{array}{l}\text { Maclura tinctoria (L.) Engl. } \\
\text { Musaceae }\end{array}$ & taiúva & CA354 & 2 & avo & quintal & $\mathrm{e}$ \\
\hline $\begin{array}{l}\text { Musa x paradisiaca } \mathrm{L} \text {. } \\
\text { Myrtaceae }\end{array}$ & banana, bananeira & il & 2 & erv & roça, quintal & $\mathrm{c}$ \\
\hline Eucalyptus sp. & eucalipto & CA308 & 5 & avo & urbano & $\mathrm{c}$ \\
\hline Eugenia uniflora $\mathrm{L}$. & pitanga & CA377 & 4 & avo & quintal & $\mathrm{c}$ \\
\hline Psidium guajava $\mathrm{L}$. & goiaba & il & 4 & avo & quintal, roça & $\mathrm{c}$ \\
\hline Syzygium cumini (L.) Skeels & jambo & CA431 & 1 & avo & quintal & $\mathrm{c}$ \\
\hline Syzygium jambos (L.) Okton & jambo & CA389 & 1 & avo & quintal & $\mathrm{c}$ \\
\hline Nyctaginaceae & & & & & & \\
\hline Boerhavia diffusa $\mathrm{L}$. & amarra-pinto, marra-pinto & $\begin{array}{l}30703 \\
30704\end{array}$ & 7 & erv & roça, quintal & $\mathrm{e}$ \\
\hline $\begin{array}{l}\text { Mirabilis jalapa } \mathrm{L} . \\
\text { Oxalidaceae }\end{array}$ & maravilha-curativa & 30702 & 4 & erv & quintal & $\mathrm{c}$ \\
\hline $\begin{array}{l}\text { Averrhoa carambola } \mathrm{L} . \\
\text { Passifloraceae }\end{array}$ & carambola & 30700 & 3 & avo & quintal & $\mathrm{c}$ \\
\hline Passiflora cincinnata Mast. & maracujá-do-mato & RS4 & 2 & $\operatorname{trp}$ & cerrado & $\mathrm{e}$ \\
\hline Passiflora edulis Sims & maracujá & il & 6 & $\operatorname{trp}$ & quintal & $\mathrm{c}$ \\
\hline
\end{tabular}


Apêndice 1. (continuação).

\begin{tabular}{|c|c|c|c|c|c|c|}
\hline Nome científico & Nome vulgar & Ref & NI & Hab & Habitat / local de coleta & $\mathbf{C} / \mathbf{E}$ \\
\hline \multicolumn{7}{|l|}{ Phytolaccaceae } \\
\hline Petiveria alliacea $\mathrm{L}$. & guiné & 30685 & 9 & erv & quintal & $\mathrm{c}$ \\
\hline \multicolumn{7}{|l|}{ Piperaceae } \\
\hline Peperomia pellucida (L.) H.B.K. & sem nome & 30769 & 1 & erv & quintal & $\mathrm{e}$ \\
\hline Piper tuberculatum Jacq. & jurandir, jaborandi & $\begin{array}{l}30669 \\
30670\end{array}$ & 6 & arb & cerrado, quintal & $\mathrm{e}$ \\
\hline Potomorphe umbellata (L.) Miq. & pariparoba & 30767 & 1 & erv & quintal & $\mathrm{e}$ \\
\hline \\
\hline Cenchrus echinatus $\mathrm{L}$. & carrapicho-de-espinho & 30712 & 1 & erv & roça & $\mathrm{e}$ \\
\hline Coix lacryma-jobi $\mathrm{L}$. & $\begin{array}{l}\text { conta-de-nosso-senhor, erva-de- } \\
\text { santa-maria, leite-de-nossa- } \\
\text { senhora }\end{array}$ & 30711 & 2 & erv & quintal & $\mathrm{c}$ \\
\hline Cymbopogon citratus Stapf. & capim-cidreira & CA300 & 10 & erv & quintal & $\mathrm{c}$ \\
\hline Eragrostis tenella (L.) Roem. \& Schult. & grama & CA347 & 1 & erv & terreno baldio & $\mathrm{e}$ \\
\hline Hyparrhenia cf. rufa (Nees) Stapf. & sapé & MC85 & 4 & erv & cerrado & $\mathrm{e}$ \\
\hline Melinis minutiflora $\mathrm{L}$. & capim-gordura & 30772 & 2 & erv & cerrado & $\mathrm{e}$ \\
\hline Saccharum officinarum $\mathrm{L}$. & cana & il & 2 & erv & roça & $\mathrm{c}$ \\
\hline Vetiveria zizanioides (L.) Nash & capim-santo & 30710 & 1 & erv & quintal & $\mathrm{c}$ \\
\hline Zea mays $\mathrm{L}$. & milho & il & 5 & erv & roça & $\mathrm{c}$ \\
\hline \multicolumn{7}{|l|}{ Polygonaceae } \\
\hline Polygonum hydropiperoides Michx. & erva-de-bicho & 30686 & 12 & erv & vargem, brejo & $\mathrm{e}$ \\
\hline Triplaris americana $\mathrm{L}$. & formigueiro & CA353 & 2 & avo & vegetação secundária & $\mathrm{e}$ \\
\hline \multicolumn{7}{|l|}{ Punicaceae } \\
\hline Punica granatum $\mathrm{L}$. & romã & il & 10 & avo & quintal & $\mathrm{c}$ \\
\hline \multicolumn{7}{|l|}{ Rhamnaceae } \\
\hline Rhamnidium elaeocarpum Reiss. & broto-de-cabrito & CA345 & 1 & avo & chácara & $\mathrm{e}$ \\
\hline \multicolumn{7}{|l|}{ Rubiaceae } \\
\hline Alibertia edulis A. Rich. ex DC. & marmelada-bola & 30784 & 1 & arb & cerrado, quintal & $\mathrm{e} / \mathrm{c}$ \\
\hline Chiococca alba Hitch. & cainca, fruta-de-urubu & 30744 & 1 & sab & vegetação secundária & $\mathrm{e}$ \\
\hline Coffea sp. & café & il & 5 & arb & quintal & $\mathrm{c}$ \\
\hline \multicolumn{7}{|l|}{ Rubiaceae (cont.) } \\
\hline \multirow[t]{3}{*}{ Palicourea rigida H.B.K. } & douradão & 30741 & 3 & arb & cerrado & $\mathrm{e}$ \\
\hline & & 30743 & & & & \\
\hline & & 30756 & & & & \\
\hline \multirow{2}{*}{ Rudgea viburnoides (Cham.) Benth. } & douradinha, erva-mulá & 30742 & 4 & arb & cerrado & $\mathrm{e}$ \\
\hline & & 30782 & & & & \\
\hline \multicolumn{7}{|l|}{ Rutaceae } \\
\hline Citrus sp. 1 & lima-de-umbigo & CA341 & 4 & avo & quintal & $\mathrm{c}$ \\
\hline Citrus sp. 2 & lima-das-peças & CA343 & 5 & avo & quintal & $\mathrm{c}$ \\
\hline Citrus $x$ aurantiifolia (Christm.) Swingle & limão-galego & CA344 & 3 & avo & quintal & $\mathrm{c}$ \\
\hline \multirow{2}{*}{$\begin{array}{l}\text { Citrus } x \text { aurantium L. var. sinensis L. e Citrus } \\
x \text { aurantium L. var. aurantium }\end{array}$} & laranja-doce, laranja-misteriosa & CA340 & 14 & avo & quintal & $\mathrm{c}$ \\
\hline & & CA342 & & & & \\
\hline \multirow[t]{2}{*}{ Citrus $x$ limon (L.) Osbeck } & limãozinho-brasileiro, limão-rosa & CA291 & 8 & avo & quintal & $\mathrm{c}$ \\
\hline & & CA339 & & & & \\
\hline Ruta graveolens $\mathrm{L}$. & arruda & il & 16 & erv & quintal & $\mathrm{c}$ \\
\hline Sapindaceae & & & & & & \\
\hline Dilodendron bipinnatum Radlk & mulher-pobre & CA403 & 2 & avo & beira de estrada & $\mathrm{e}$ \\
\hline Paullinia cupana H.B. K. & guaraná & - & 7 & arb & $*$ & \\
\hline Scrophulariaceae & & & & & & \\
\hline Angelonia salicariaefolia Humb. \& Bonpl. & atrativo & 30731 & 1 & erv & quintal & $\mathrm{c}$ \\
\hline cf. Angelonia hirta Cham. & pronto-alívio & CA26 & 1 & erv & vargem, brejo & $\mathrm{e}$ \\
\hline Scoparia dulcis L. & vassourinha & 30727 & 14 & erv & terreiro & $\mathrm{e}$ \\
\hline & & 30728 & & & & \\
\hline Simaroubaceae & & & & & & \\
\hline Simaba trichilioides St.Hil. & calunga, calonga & 30722 & 7 & sab & cerrado & $\mathrm{e}$ \\
\hline Simarouba versicolor St.Hil. & pau-de-perdiz & CA263 & 1 & avo & cerrado & $\mathrm{e}$ \\
\hline Solanaceae & & & & & & \\
\hline Capsicum annuum $\mathrm{L}$. & pimentão & il & 1 & $\mathrm{sab}$ & roça de beira do rio & $\mathrm{c}$ \\
\hline Capsicum frutescens $\mathrm{L}$. & pimenta, pimenta-malagueta & il & 5 & $\mathrm{sab}$ & quintal & $\mathrm{c}$ \\
\hline Lycopersicon esculentum Mill. & tomate & il & 1 & erv & quintal & $\mathrm{c}$ \\
\hline Nicotiana tabacum $\mathrm{L}$. & fumo & il & 5 & erv & roça de beira do rio, $*$ & $\mathrm{c}$ \\
\hline Solanum americanum Mill. & carichichu & 30724 & 1 & erv & quintal & $\mathrm{e}$ \\
\hline Solanum cf. comptum Morton & joá & 30719 & 3 & $\mathrm{sab}$ & cerrado, brejo & $\mathrm{e}$ \\
\hline Solanum cf. variabile Mart. & jurubeba & 30721 & 1 & arb & quintal & $\mathrm{c}$ \\
\hline Solanum gilo Raddi & jiló & - & 1 & erv & $*$ & \\
\hline
\end{tabular}


Apêndice 1. (continuação).

\begin{tabular}{|c|c|c|c|c|c|c|}
\hline Nome científico & Nome vulgar & Ref & NI & Hab & Habitat / local de coleta & $\mathbf{C} / \mathbf{E}$ \\
\hline \multicolumn{7}{|l|}{ Sterculiaceae } \\
\hline Byttneria melastomifolia A. St.Hil. & raiz-de-bugre & $\begin{array}{l}30697 \\
30698\end{array}$ & 12 & erv & cerrado & $\mathrm{e}$ \\
\hline Guazuma ulmifolia Lam. & chico-magro & $\begin{array}{l}30726 \\
30720\end{array}$ & 2 & avo & cerrado, terreiro & $\mathrm{e}$ \\
\hline Helicteres sacarolha A. St.Hil. & rosquinha, rosquinha-de-gato & $\begin{array}{l}30694 \\
30695\end{array}$ & 6 & $\mathrm{sab}$ & cerrado & $\mathrm{e}$ \\
\hline \multicolumn{7}{|l|}{ Verbenaceae } \\
\hline Lantana camara $\mathrm{L}$. & cambará & 30691 & 1 & $\mathrm{sab}$ & terreno baldio & $\mathrm{e}$ \\
\hline Lippia alba (Mill.) N.E.Br. & $\begin{array}{l}\text { erva-cidreira, cidreira de folha, } \\
\text { melissa-de-casa }\end{array}$ & $\begin{array}{l}\text { CA407 } \\
\text { CA408 }\end{array}$ & 8 & $\mathrm{sab}$ & quintal & $\mathrm{c}$ \\
\hline Stachytarpheta cayenensis (L.C. Rich.) Vahl. & gerbão, jorbão & $\mathrm{MC} 2$ & 11 & $\mathrm{sab}$ & quintal & $\mathrm{e}$ \\
\hline $\begin{array}{l}\text { Vitex cymosa Bert. } \\
\text { Vitaceae }\end{array}$ & tarumã, tarumeiro & 30690 & 3 & avo & terreiro & $\mathrm{e} / \mathrm{c}$ \\
\hline $\begin{array}{l}\text { Cissus verticillata (L.) Nicolson \& Jarvis } \\
\text { Vochysiaceae }\end{array}$ & insulina & CA336 & 1 & $\operatorname{trp}$ & quintal & $\mathrm{c}$ \\
\hline Callisthene fasciculata (Spreng.) Mart. & carvão-branco & 30845 & 6 & avo & baixada, beira de rio & $\mathrm{e}$ \\
\hline Qualea grandiflora Mart. & pau-terra-macho & 30848 & 3 & avo & cerrado & $\mathrm{e}$ \\
\hline cf. Salvertia convalariodora A. St.Hil. & pau-doce & CA256 & 2 & avo & cerrado & $\mathrm{e}$ \\
\hline Vochysia divergens Pohl. & cambará & 30846 & 5 & avo & cerrado, próximo de vargem & $\mathrm{e}$ \\
\hline Zingiberaceae & & & & & & \\
\hline Alpinia zerumbet (Pers.) Burtt \& Smith & colonia & 30693 & 15 & erv & quintal & $\mathrm{c}$ \\
\hline Curcuma sp. & açafrão & CA326 & 3 & erv & quintal & $\mathrm{c}$ \\
\hline Hedychium coronarium Koen. & palma-de-são-josé & 30692 & 1 & erv & quintal & $\mathrm{e} / \mathrm{c}$ \\
\hline
\end{tabular}

